\title{
Clinicopathological and Immunohistochemical Profile of Mantle Cell Lymphoma: An Institutional Experience
}

\author{
Pritinanda Mishra ${ }^{1}$, Somanath Padhi ${ }^{1}$, Pavithra Ayyanar ${ }^{1}$, Swagatika Samal ${ }^{1}$, Saroj Das Majumdar ${ }^{2}$, \\ Ashutosh Panigrahi ${ }^{3}$, Mukund Sable ${ }^{1}$ \\ 1. Pathology, All India Institute of Medical Science (AIIMS), Bhubaneswar, IND 2. Radiation Oncology, All India \\ Institute of Medical Science (AIIMS), Bhubaneswar, IND 3. Hematology and Medical Oncology, All India Institute of \\ Medical Science (AIIMS), Bhubaneswar, IND
}

Corresponding author: Mukund Sable, pathol_mukund@aiimsbhubaneswar.edu.in

\section{Abstract \\ Introduction \\ Mantle cell lymphoma (MCL) is a biologically aggressive B-cell non-Hodgkin lymphoma (NHL) with distinctive morphologic, immunophenotypic, and molecular characteristics. Differentiation from other chronic lymphoproliferative disorders is essential for prognostication.}

\section{Aim}

This paper aims to study the clinicopathological features of MCL with emphasis on immunohistochemical features and disease correlation.

\section{Method}

To do so, clinicopathological characteristics from 21 cases of MCL (14 males, seven females, M:F=2:1) diagnosed in the last five years i.e. 2015 to 2020, were retrospectively reviewed and correlated with immunohistochemistry (IHC) data. Particularly those pertaining to cyclin D1, SRY-box transcription factor 11 (SOX11), cluster of differentiation (CD) 5, CD23, MIB E3 ubiquitin protein ligase 1 (MIB1), tumor protein 53 (TP53), c-myelocytomatosis oncogene product (c-MYC), multiple myeloma oncogene 1 (MUM1), mouse double minute 2 homolog (MDM2), and Epstein-Barr virus latent membrane protein 1 (EBV-LMP1) expression with its aberrations.

\section{Observations}

This study shows that MCL constituted 4.2\% (21/500) of all NHLs with a mean age of 57.5 years (median 60 years, range 30 to 80 years). The disease was nodal in 19 , and extranodal in the remaining two cases. 14 of 21 (67\%) had generalized lymphadenopathy and $71 \%$ had bone marrow (BM) involvement. The nodal involvement was diffuse in 9/17 (53\%), 8/21 (38\%) had a blastoid morphology, and an in-situ MCL pattern was not seen in any of the cases selected for the study. Cyclin D1 immunoexpression correlated well

Review began 05/26/2021 Review ended 07/07/2021 Published 07/21/2021

๑) Copyright 2021

Mishra et al. This is an open access article distributed under the terms of the Creative Commons Attribution License CC-BY 4.0., which permits unrestricted use, distribution, and reproduction in any medium, provided the original author and source are credited. with SOX11; CD5-negative in five cases; and CD23-positive in three cases. TP53 and c-MYC expression were noted in 17/19 (89.4\%) and 8/17 (47\%), respectively. MUM1 registered positive in six cases. None of the cases showed immunopositivity for MDM2 and EBV-LMP1.

\section{Conclusion}

In essence, this study indicates that morphological and immunophenotypic subclassification of mantle cell lymphoma with a wider panel of IHC markers is essential for understanding disease biology and better prognostication.

Categories: Pathology, Hematology

Keywords: mantle cell lymphoma, cyclin d1, sox11, c-myc, tp53, aberrant phenotype, biology

\section{Introduction}

Mantle cell lymphoma (MCL) is a relatively rare lymphoproliferative neoplasm (LPN), accounting for less than $10 \%$ of all non-Hodgkin lymphomas (NHL) [1]. Its morphology is quite homogeneous, but it varies strikingly in about $10 \%$ of the cases, making the diagnosis of MCL challenging for histopathologists. The disease is characterized by hallmark translocation $\mathrm{t}(11 ; 14)$ (q13; q32) which juxtaposes with cyclin D1 and the immunoglobulin heavy chain genes resulting in an increased expression of the cyclin D1 protein, which in turn results in cellular proliferation and increased survival [2]. Although morphology and immunohistochemical features [B-lymphocyte antigen cluster of differentiation (CD)20 or CD20-positive(+)/ CD5+/ cyclin D1+/ CD23-negative(-)/ CD10(-)] are characteristic for diagnosis, aberrant phenotypic 
expressions are not uncommon [3], and these are more commonly reported in blastoid phenotype [4]. Common aberrancies reported in the literature (either in isolation or in combinations) include CD5-, CD23+, CD10+, B-cell lymphoma 6 protein or BCL6+, and cyclin D1- subgroups that may pose a diagnostic dilemma for the surgical pathologist [4-6].

Recently, c-myelocytomatosis oncogene product (c-MYC) and tumor protein 53 (TP53) gene rearrangement were reported to be associated with aggressive biological behavior and overall inferior survival in a subgroup of MCL subjects for which, further in-depth studies are necessary [7,8]. The objective of our present study is to discern the immunophenotypic characteristics of a cohort of MCL cases on lymph node (LN) biopsies and compare them with clinicopathological features and their impact on disease biology. We also present a summary of the relevant published literature pertinent to our observations.

\section{Materials And Methods}

The archival biopsy material from the surgical pathology section of the Department of Pathology in a tertiary care center based in eastern India was searched retrospectively for cases of MCL diagnosed over the last five years (2015 to 2020). We included both nodal and extranodal cases in our series, and their morphological and immunohistochemistry (IHC) characteristics were reviewed and analyzed by two independent surgical pathologists (PNM, MNS) as per the criteria proposed by the 2015 World Health Organization (WHO) classification of hematopoietic and lymphoid neoplasm [4]. We included 21/28 MCL cases following stringent criteria where detail morphological and immunophenotypical data of lymph node and bone marrow (BM) biopsy were available for descriptive study. The remaining $7 / 28$ cases in the leukemic phase, diagnosed purely based on peripheral smear and BM morphology with a limited panel of IHC, were excluded as LN biopsies were not performed in those, and flow cytometry immunophenotype data were not available in most of them.

Data pertaining to age, gender, lymphadenopathy, organomegaly, peripheral blood, and BM involvement were collected from medical records. Four microns thick deparaffinized and hematoxylin and eosin (H\&E) stained tissue sections were subjected to routine morphological analysis describing the following features: nodal architecture, the morphological phenotype of tumor cell (classical vs. blastoid), angiocentricity, proliferation index [per 10 high power (x400) fields], presence or absence of extranodal spread, high endothelial venules (HEV), pink histiocytes, residual follicles.

The following panel of antibodies was used for further characterization as per protocol with pre-treatment by microwave heating: CD20 (Clone L26, Pathnsitu, 1:100), CD3 (polyclonal, Pathnsitu, 1:100), CD5 [Clone 4C7, Dako, ready to use (RTU)], CD10 (Clone 56Cb, Dako, RTU), CD23 (Clone DAK CD23, Dako, RTU), cyclin D1 (Clone EP12, Dako, RTU), BCL2 (Clone EP36, Pathnsitu, 1:100), BCL6 (polyclonal, Biocare, RTU), TdT (Clone EP299, Pathnsitu, RTU), MIB1 (Dako, 1:150), SOX11 (Clone MRQ58, Cell marque, RTU), TP53 (Clone BP53-12, Pathnsitu, RTU), c-MYC (Clone EP121, Pathnsitu, RTU), MDM2 (Clone SMP14, Sigma-Aldrich, 1:1000), MUM-1 (Clone EP190, Pathnsitu, RTU) and EBV-LMP1 (Clone CS1-4, Dako, RTU).

We followed the cut-off as proposed by Ribera-Cortada et al. for SOX11; $(\geqslant 10 \%$ tumor cells with nuclear expression as positive) [9]. Furthermore, TP53 and c-MYC expression were semi quantified by a histoscore (HScore) calculated by multiplying proportion of positivity [0; negative, $1($ low + ); $1-10 \%+, 2$ (intermediate+); 11-50\%+, and 3 (high+) (>50\%+)] with intensity [1+; weak, 2+; intermediate, $3+$; strong]. MDM2 and MUM1 (both nuclear) and EBV-LMP1 (membrane) results were reported as positive or negative. Follow-up data was obtained from telephonic conversations with patients or their next of kin. Overall survival (OS) period was calculated from the date of diagnosis to the date of death or last follow-up.

\section{Statistical analysis}

All categorical variables were expressed in frequency and percentage. OS was compared between two subgroups in relation to age ( $\leqslant 60$ vs. > 60 years), pattern of nodal involvement (diffuse vs. non-diffuse), variant (classical vs. blastoid), MIB1 index ( $30 \leqslant$ vs. > 30\%), TP53 and c-MYC expression (high/intermediate vs. low/negative), and SOX11 (positive vs. negative). The p-value $<0.05$ was considered statistically significant. All statistical analyses were done on IBM SPSS (IBM Corp. Released 2015. IBM SPSS Statistics for Windows, Version 23.0. Armonk, NY: IBM Corp.).

\section{Results}

\section{Clinicopathological features}

The clinicopathological characteristics, immunohistochemical profile of all 21 cases of MCL are presented in the Tables below. These constituted $4.2 \%$ of all NHLs (21/500) diagnosed over the last five years at our center. There were 14 males and seven females (M:F=2:1) with a median age at diagnosis of 60 years (range 30 to 81 years). Generalized lymphadenopathy with associated hepatosplenomegaly was noted in 14 (66.7\%) and seven (33.3\%) of the cases, respectively. Two had extranodal involvement (stomach, caecum, one each). One (case no. 16) had multiple nodular skin lesions (Figure 1) in addition to generalized lymphadenopathy, splenomegaly, and bone marrow involvement. And 15 cases (71.4\%) (including positron emission tomography or PET scan finding of one case) had BM involvement at the time of initial diagnosis (Table 1). 


\section{Cureus}

\begin{tabular}{|c|c|c|c|c|c|c|c|c|c|c|}
\hline \multirow{2}{*}{$\begin{array}{l}\text { Case } \\
\text { no }\end{array}$} & \multirow{2}{*}{$\begin{array}{l}\text { Age }(y) / / \\
\text { Gender }\end{array}$} & \multirow{2}{*}{ Site } & \multirow{2}{*}{$\begin{array}{l}\text { Generalized } \\
\text { LN }\end{array}$} & \multirow{2}{*}{ HSM } & \multicolumn{3}{|c|}{ Peripheral smear findings } & \multirow{2}{*}{$\begin{array}{l}\text { BM aspiration \% of atypical } \\
\text { lymphoid cells }\end{array}$} & \multirow{2}{*}{ BMBx } & \multirow{2}{*}{$\begin{array}{l}\text { Follow up } \\
\text { (months) }\end{array}$} \\
\hline & & & & & $\begin{array}{l}\text { TLC } \\
\text { (/cu.mm) }\end{array}$ & $\begin{array}{l}\text { ALC } \\
\text { (/cu.mm) }\end{array}$ & $\begin{array}{l}\text { Atypical LC } \\
(\%)\end{array}$ & & & \\
\hline 1 & $81 / \mathrm{M}$ & Epitrochlear & Present & Splenomegaly & 21,000 & 4200 & 25 & 50 & Involved & Dead, 2 \\
\hline 2 & $52 / \mathrm{M}$ & Cervical & Present & Splenomegaly & 15,000 & 2250 & 10 & 22 & Involved & Dead, 34 \\
\hline 3 & $54 / \mathrm{M}$ & Axillary & Present & HSM & NA & NA & NA & NA & NA & Dead, 10 \\
\hline 4 & $61 / \mathrm{F}$ & Epitrochlear & Absent & Absent & 9000 & 2700 & 00 & 00 & $\begin{array}{l}\text { Not } \\
\text { involved }\end{array}$ & Alive, 37 \\
\hline 5 & $49 / M$ & Cervical & Present & Absent & NA & NA & NA & NA & $\begin{array}{l}\text { Involved } \\
\neq\end{array}$ & Dead, 21 \\
\hline 6 & $65 / F$ & Cervical & Present & Absent & 10390 & 2078 & 14 & 50 & Involved & NA \\
\hline 7 & $66 / \mathrm{M}$ & Cenvical & Present & Absent & 12130 & 4852 & 35 & 30 & Involved & Dead, 3 \\
\hline 8 & $61 / \mathrm{M}$ & Gastric & Absent & HSM & 13000 & 1560 & LEB,12 & Dry tap & Involved & Dead, 6 \\
\hline 9 & $71 / \mathrm{F}$ & Supraclavicular & Present & Absent & 8700 & 1740 & 10 & 15 & Involved & Dead, 8 \\
\hline 10 & $60 / M$ & Inguinal & Absent & Absent & 7900 & 1940 & 00 & NA & NA & NA \\
\hline 11 & $64 / \mathrm{M}$ & Caecal growth & Absent & Absent & NA & NA & NA & NA & NA & NA \\
\hline 12 & $57 / F$ & Axillary & Present & Splenomegaly & 8600 & 4386 & 10 & 30 & Involved & Alive, 30 \\
\hline 13 & $45 / \mathrm{F}$ & Inguinal & NA & NA & NA & NA & NA & NA & NA & NA \\
\hline 14 & 70/F & Inguinal & Absent & Absent & 8720 & 1744 & 05 & 25 & Involved & Alive, 46 \\
\hline 15 & $47 / M$ & Cervical & Present & NA & NA & NA & NA & NA & NA & Alive, 18 \\
\hline 16 & $40 / F$ & $\begin{array}{l}\text { Cervical, skin } \\
\text { lesion }\end{array}$ & Present & Splenomegaly & 10630 & 2890 & 05 & 80 & Involved & Dead, 16 \\
\hline 17 & $30 / \mathrm{M}$ & Cervical & Present & Splenomegaly & 18510 & 14,580 & 75 & 40 & Involved & Alive, 10 \\
\hline 18 & $50 / M$ & Supraclavicular & Absent & Absent & 52980 & 43530 & 35 & 35 & Involved & Dead, 3 \\
\hline 19 & 50/M & Inguinal & Present & Absent & 14850 & 3118 & 15 & 10 & Involved & Alive, 6 \\
\hline 20 & $60 / M$ & Cervical & Present & Absent & 7810 & 1405 & 00 & 05 & Involved & Alive, 3 \\
\hline 21 & $74 / \mathrm{M}$ & Inguinal & Present & Absent & 14000 & 4620 & 26 & 30 & Involved & Alive, 2 \\
\hline
\end{tabular}

\section{TABLE 1: Clinicohematological profile of 21 patients of MCL.}

Abbreviations: ALC: absolute lymphocyte count, BM: bone marrow, BMBx: bone marrow biopsy, F: female, HSM: hepatosplenomegaly, M: male, LN: Iymphadenopathy, LC: lymphoid cell, NA: not available, TLC: total leucocyte count.

\#PETCT showed F-fluorodeoxyglucose (FDG) uptake in the pelvic bones.

Histomorphological and immunohistochemical details of cases with extranodal involvement (case no.11 and case no.16) are described in Figure 1. 


\section{Cureus}

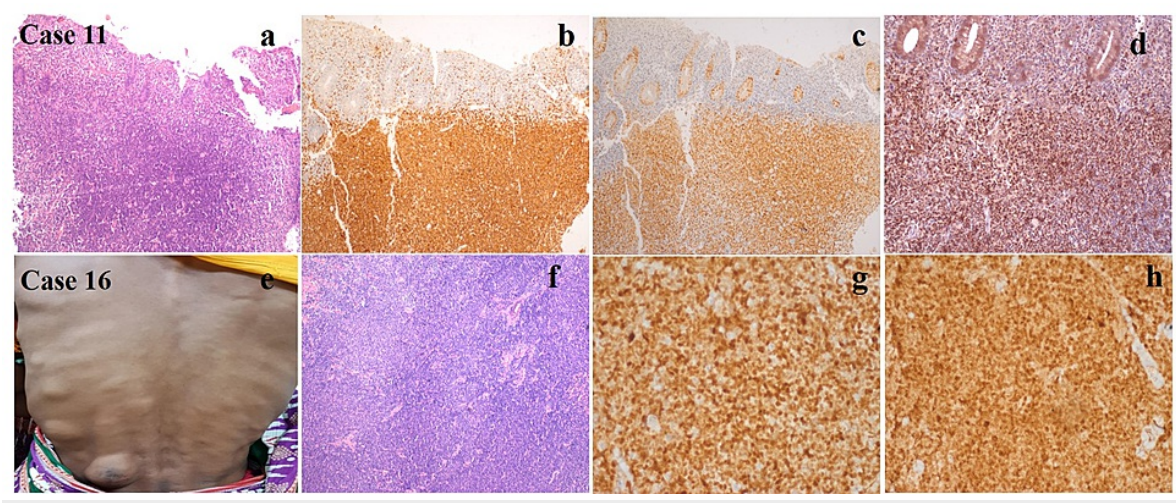

FIGURE 1: (a) Fragments of colonic mucosa showing infiltration by atypical lymphoid cells H\&E 100X, (b) Lymphoid cells are immunopositive for CD5, (c) Cyclin D1, (d) TP53, (e) Skin involvement in MCL presenting as subcutaneous nodules, (f) Classical small cell morphology of MCL. H\&E 100X, (g) Lymphoid cells are immunopositive for cyclin D1, (h) SOX11 (IHC X 200X)

\section{Histopathological findings}

Excision lymph node biopsy material was available in 17 of the cases, needle core LN biopsy in two, and mucosal biopsies in two cases (as above) for histopathological examination (Table 2). 


\section{Cureus}

\begin{tabular}{|c|c|c|c|c|c|c|c|c|c|}
\hline & Pattern & In-situ lesion & Pink histiocytes & HEV & Angiocentricity & Residual follicles & Mitosis/10HPF & ENE & Morphological types \\
\hline 1 & Diffuse & - & Present & Present & Present & Present & 26 & Present & Blastoid \\
\hline 2 & Diffuse & - & Present & Present & Present & Present & 15 & Present & Classical \\
\hline 3 & Diffuse & - & Present & Present & Present & Absent & 14 & Absent & Classical \\
\hline 4 & Diffuse & - & Present & Present & Present & Present & 65 & Present & Blastoid \\
\hline 5 & Nodular & - & Absent & Absent & Present & Present & 15 & Present & Classical \\
\hline 6 & Diffuse & - & Present & Absent & Present & Present & 09 & Present & Classical \\
\hline 7 & Mixed & - & Present & Present & Present & Absent & 78 & Present & Blastoid \\
\hline 8 & Diffuse & - & Absent & Absent & Present & NA & 10 & NA & Classical \\
\hline 9 & Mixed & - & Absent & Absent & Present & Absent & 28 & Present & Blastoid \\
\hline 10 & Diffuse & - & Present & Absent & Present & Present & 10 & Present & Classical \\
\hline 11 & Diffuse & - & Absent & Absent & Absent & NA & 10 & NA & Classical \\
\hline 12 & Nodular & - & Present & Present & Present & Present & 10 & Present & Classical \\
\hline 13 & Diffuse & - & Present & Present & Present & NA & 75 & NA & Blastoid \\
\hline 14 & Mixed & - & Present & Present & Present & Absent & 16 & Present & Classical \\
\hline 15 & Diffuse & - & Present & Present & Present & Present & 10 & Present & Classical \\
\hline 16 & Mixed & - & Present & Present & Present & Absent & 05 & Present & Classical \\
\hline 17 & Mixed & - & Present & Absent & Absent & Absent & 60 & Present & Blastoid \\
\hline 18 & Diffuse & - & Present & Present & Present & Absent & 16 & Present & Classical \\
\hline 19 & Diffuse & - & Absent & Absent & Absent & NA & 50 & NA & Blastoid \\
\hline 20 & Diffuse & - & Present & Present & Present & Absent & 14 & Present & Classical \\
\hline 21 & Nodular & - & Present & Present & Present & Present & 35 & Present & Blastoid \\
\hline
\end{tabular}

TABLE 2: Histopathological findings in 21 patients of $M C L$

Abbreviations: NA: not available, HEV: high endothelial venules, HPF: high power field, ENE: extranodal extension

Morphologically, the neoplastic lymphoid cells showed a classical small cell morphology with irregular nuclear outline in 13/21 (62\%) cases (classical variant); and the remaining eight (38\%) cases had a blastoid phenotype with prominent nucleoli and increased mitoses. Pleomorphic variant of MCL was not encountered in any of our cases. Capsular infiltration, tumor angiocentricity; and pink histiocytes were described in 16/17 (94\%), 18/21 (85\%), and 16/21 (76\%) cases, respectively.

\section{Immunohistochemical findings}

Classical immunophentype such as CD20-positive/ CD5-positive/ CD10-negative/ CD23-negative/ cyclin D1positive/ SOX11-positive was seen in 16 cases (76.2\%), whereas aberrant phenotype was noted in five (23.8\%) (Table 3). 


\section{Cureus}

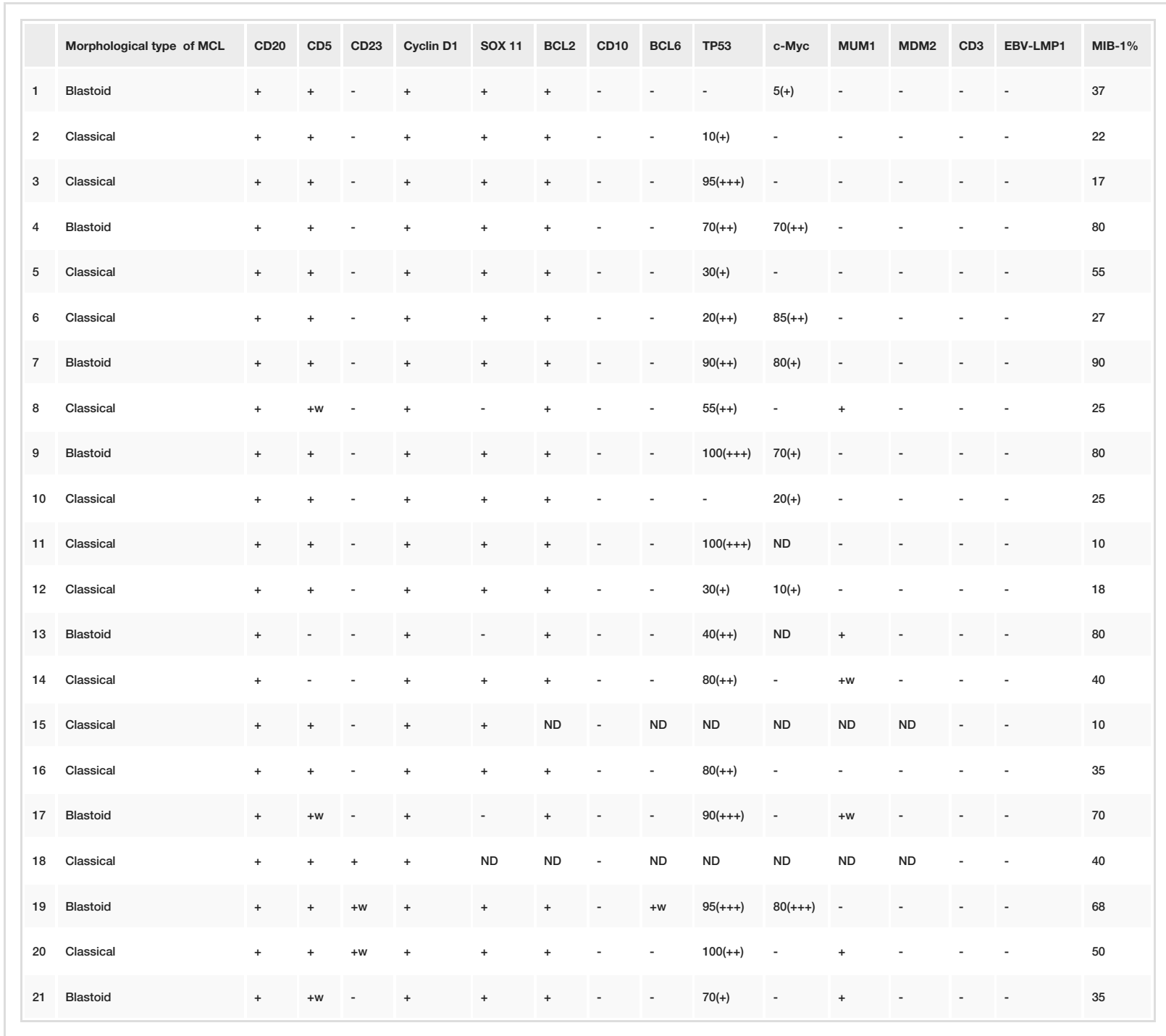

\section{TABLE 3: Immunohistochemical profile of 21 patients of MCL}

Abbreviations: CD: cluster of differentiation, ND: not done, -: negative, + : positive, + w: weakly positive, Intensity : $(+)=$ weak, $(++)=i n t e r m e d i a t e$, $(+++)=$ strong

Common aberrancies observed were as follows: CD5 (negative to weak + ); $\mathrm{n}=5$ (three blastoid, two classical), SOX11-negative; $\mathrm{n}=3$ (two blastoids), CD23+/weak+; $\mathrm{n}=3$ (two classical, one blastoid), BCl6weak+; $\mathrm{n}=1$ (blastoid). While cyclin D1 expression was noted in all cases (21/21, 100\%), TP53 expression was noted in $17 / 21$ (80.9\%) cases (seven with blastoid phenotype, high/strong in five, intermediate in eight, weak in four); and MUM1-positive was noted in 6/19 cases (31.5\%). Similarly, c-MYC expression was noted in 8/17 (47\%) cases [high to intermediate in three (two blastoids), and weak in five]. The mean MIB1 proliferation rate of classical and blastoid subgroups were $28.8 \%$ and $67.5 \%$, respectively. MDM2 and EBV-LMP1 were negative in all cases. Histomorphological and immunohistochemical details of aberrant cases (case no.17 and case no.20) are described in Figure 2. 


\section{Cureus}

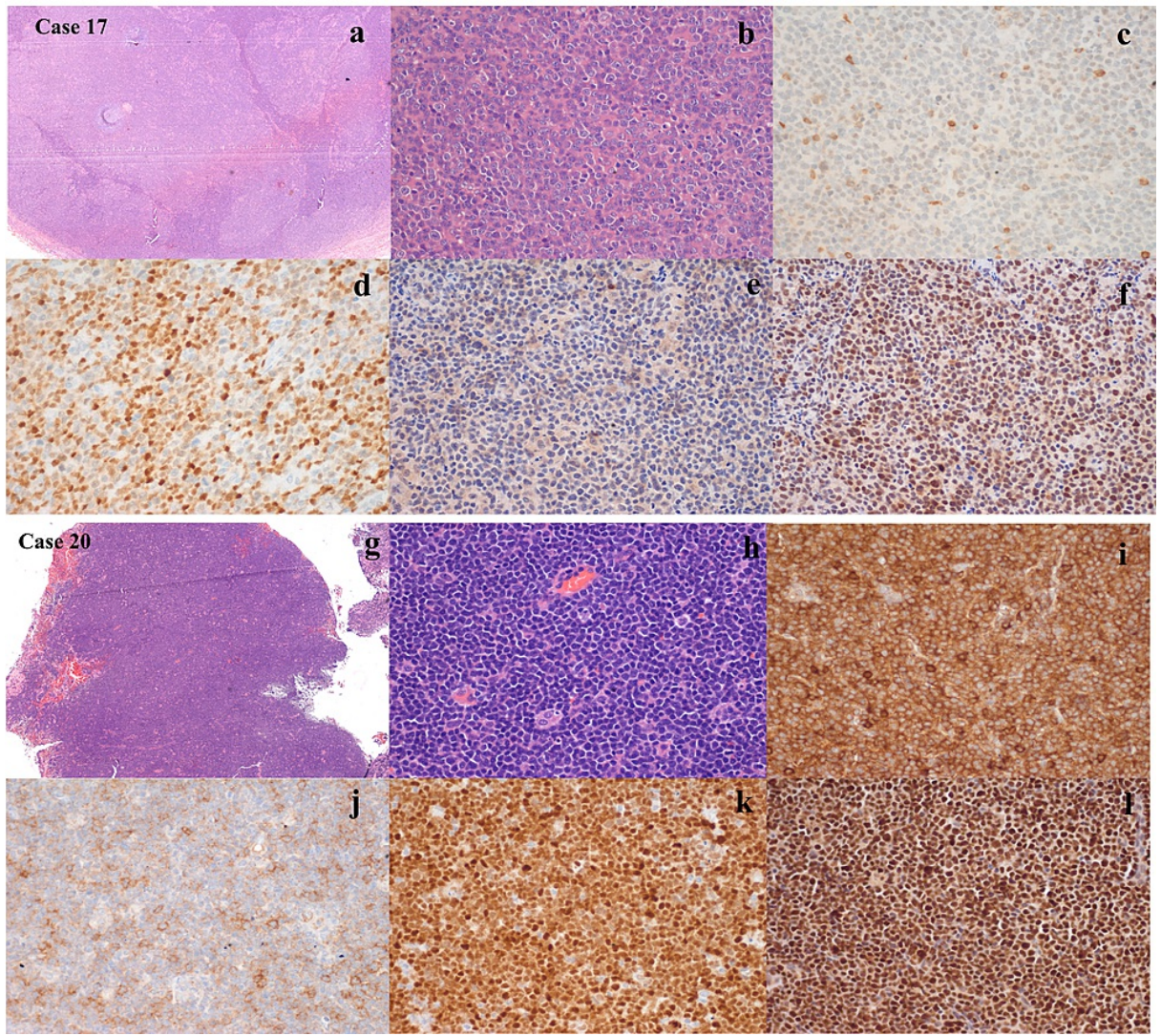

FIGURE 2: (a) Nodular effacement of the lymph node, H\&E 40X, (b) Atypical lymphoid cells are large with prominent nucleoli, blastoid morphology. H\&E 200X, (c) Lymphoid cells are immunonegative for CD5, (d) Immunopositive for cyclin D1, (e) Immunonegative for SOX11, (f) Immunopositive for TP53. (IHC X200), (g) Diffuse infiltration of atypical lymphoid cells. H\&E 40X, (h) Prominence of pink histiocytes. H\&E 100X, (i) Lymphoid cells are immunopositive for CD5, (j) Immunopositive for CD23, (k) Immunopositive for cyclin D1, (I) Immunopositive for SOX11. IHC X 200

None of the cases showed immunoprofile as CD5-negative/CD23-positive/SOX11-negative.

\section{Hematological evaluation}

Complete blood counts and BM data were available in 16 cases and 15 cases, respectively. Circulating neoplastic lymphoid cells were evident in $13 / 16$ (81\%) which ranged from $5 \%$ to $75 \%$ of differential leukocyte count (median=14\%). 6/8 (75\%) blastoid MCL had peripheral blood spillage compared to 7/13 (53.8\%) classical variant. BM involvement was reported in 14/15 (93.3\%) cases with the lymphoma cells comprising of $5 \%$ to $80 \%$ of marrow nucleated cells (median $=15$ ) in aspirate smears (case no. 5 showed Ffluorodeoxyglucose (FDG) uptake in the pelvic bones, and bone marrow examination was not available). The pattern of BM involvement in Table 1 was reported as follows: nodular $(n=5)$, paratrabecular $(n=2)$, mixed $(\mathrm{n}=5)$, diffuse $(\mathrm{n}=2)$.

\section{Follow-up and overall survival in various prognostic subgroups}

The median duration of follow-up post-therapy ( $\mathrm{n}=17 / 21$ ) was 10 months (range two to 46 months); with nine (52.9\%) dead at the time of the last follow-up. The overall survival did not vary between two subgroups in regard to age ( $60 \leqslant$ vs. $>60$ years, 29.6 vs. 22.3 months, respectively, log-rank $\mathrm{P}=0.067$ ), morphology (classic vs. blastoid, 23.72 vs. 19.54 months, respectively, log-rank $\mathrm{P}=0.769$ ), TP53 expression (high vs. weakintermediate, 22.23 vs. 29.66 months, respectively, log-rank $\mathrm{P}=0.604$ ), c-MYC expression (positive vs. negative, 19.05 vs. 23.1 months, respectively, log-rank $\mathrm{P}=0.881$ ), SOX11 (positive vs. negative, 25 vs. 8.0months, respectively, $\log$-rank $\mathrm{P}=0.636$ ); and proliferation rate (low vs. high, 22.3 vs. 23.0 months, respectively, log-rank $\mathrm{P}=0.961$ ). 


\section{Discussion}

We studied 21 cases of MCL diagnosed over a period of five years that occurred in the elderly age group with advanced disease at presentation. The prevalence in our series $(4.2 \%)$ was slightly more than two other Indian series where it was reported to be $2.1 \%$ and $3.4 \%$, respectively $[1,10]$. Compared to a large series by Hrgovic et al. where 22 cases of cutaneous involvement by MCL was reported, we found such occurrence in one case only and the other two had focal gastrointestinal involvement $[4,11]$. Classical morphology dominated the blastoid phenotype (13 vs. eight, respectively). Immunophenotypic aberrancies such as CD5negative, CD23-positive, SOX11-negative were noted (9.5\%, $14.3 \%$, and $14.3 \%$ cases respectively), and TP53 and c-MYC expression were observed in a good number/higher proportion of our cases. However, none of the clinicopathological characteristics impacted the overall survival.

The pattern of nodal histomorphology in MCL varies from diffuse to nodular-diffuse or a mantle cell pattern. From a morphological point of view, the greatest challenge is to differentiate the latter from its precursor state, the so-called 'In-situ mantle cell neoplasia (ISMCN)'. ISMCN is characterized by preserved nodal architecture with reactive follicles without an expanded mantle, which characteristically harbors cyclin D1positive cells confined to the inner zone in some of the follicles. In contrast, the MCL with a mantle cell pattern could be recognized by its more numerous and crowded follicles with a decreased interfollicular area and expanded mantle zone, and the zone showing diffuse nuclear positivity for cyclin D1. The mantle cell pattern of MCL was not seen in our series. Though the 'in-situ MCL is an incidental finding, its pathological significance remains that these may represent the precursor stage of lymphomagenesis with similar molecular signature and confer a favorable prognosis [12]. We did not find any evidence of 'in-situ lesion' in our cases; a feature pointing towards an advanced disease with an increased propensity for peripheral blood spillage.

The blastoid variant exhibits large cells with nucleoli and a very brisk mitotic activity (>20-30/10 HPF) and sometimes may mimic a lymphoblastic lymphoma. Cyclin D1 positivity and absence of terminal deoxynucleotidyl transferase (TdT) helps in differentiating the two. The nodular pattern of involvement of the nodes allows other low-grade lymphomas like chronic lymphocytic leukemia (CLL) or follicular lymphoma (FL) into the differentials. The absence of nuclear indentations, presence of pro-lymphocytes, and proliferation centers negate MCL. A mixture of cleaved and non-cleaved cells in a follicular pattern favor FL. In addition, pink histiocytes, high endothelial venules, and angiocentric patterns of atypical lymphoid cells are subtle clues for the diagnosis of MCL [13]. Though they are morphological clues for aiding the diagnosis, their prognostic significance is questionable.

Immunophenotypic aberrations in MCL have been described in the literature $[3,5,6]$. This possibly results due to origin from the germinal center and/or post germinal center B lymphocytes through the somatic hypermutation of the immunoglobulin heavy-chain variable region (IgVH), especially IGHV3-21, IGHV3-23, IGHV4-34, and IGHV4-59 [14].

Classical immunophenotype of MCL is CD20-positive/ CD5-positive/ cyclin D1-positive/ CD23-negative/ CD10-negative. Though cyclin D1 is one of the most persistent nuclear markers for MCL, it can be absent in a subset of lymphoma known as cyclin D1-negative MCL. This subgroup was clinically aggressive with advanced stage at diagnosis with frequent peripheral blood and extranodal involvement; thus a poor response to therapy [15]. Hence, it is important to diagnose this entity, so that other treatment options could be offered in these groups of MCL. In our series, cyclin D1 was universally expressed by MCL cells; though blastoid phenotype had heterogeneous nuclear positivity compared to stronger intensity among classical subgroup [16].

The largest series by Miao et al. has shown 6\% absence of CD5 expression [17] and in the present study, CD5 was not seen in $9 \%$ of cases (2/21) [Blastoid (1), Classical (1)], though weak expression was observed in $14 \%$ of cases (3/21). Shih et al. showed that all CD5-negative MCL have a classical morphology [18]. A plausible explanation for these differences could possibly be attributed to the lesser number of cases included in the study groups. CD23 is frequently negative in MCL. A study by Saksena et al. has shown that $13 \%$ of MCL cases showed CD23 expression and these cases are associated with leucocytosis, a leukemic presentation, bone marrow involvement, CD200 expression, and a lower frequency of SOX11 positivity [19]. In the present study, $14 \%$ (3/21) of cases showed membrane positivity for CD23 (one strong expression and two weak expressions) and $66.6 \%$ of patients had an elevated leukocyte count and all of them showed bone marrow involvement.

SOX11 immuno-expression was absent in three of our cases (15\%) (two blastoid phenotypes). Xu et al. described a large series $(n=75)$ of SOX11-negative MCL and reported that such subgroups have classic morphology, increased CD23 coexpression, lower proliferation rate, increased extranodal involvement, and more propensity for leukemic transformation compared to the SOX11-positive subgroup [20]. Similar observations were also made by Nygren et al. who found that the SOX11-negative group has significantly lower OS compared with SOX11-positive cases (median OS: 1.5 vs. 3.2 years, respectively; $\mathrm{P}=0.014$ ) [21]. In contrast, the SOX11-negative phenotype offered a significantly better survival advantage over the positive subgroup (five-year OS: $78 \%$ vs. $38 \%$, respectively, $\mathrm{P}=0.001$ ) in another study by Fernandez et al. [22]. 
In previous studies, the percentages of MUM1 immunoexpression in MCL ranged from 11\% to 36\% [23,24]. The present study showed positivity in $28 \%$ of cases. Though MDM2 expression is associated with poor prognosis in MCL, our study shows that all cases of MCL were immunonegative for MDM2 [25]. Similarly, very few reports showed immunopositivity for EBV-LMP1 [26].

Strong immunoexpression of TP53 and c-MYC in MCL are usually seen with blastoid and pleomorphic morphology and are associated with aggressive behavior and poor outcome. None of the classical cases showed a strong intensity of c-MYC or TP53 staining [16]. However, in contrast to previous observations, the present study reveals the strong immunoexpression of TP53 in two cases of classical MCL and intermediate immunoexpression in five cases of classical MCL.

\section{Conclusions}

We studied the clinicopathological and immunohistochemical features of MCL in our population. Also, the aberrant phenotypic alterations and their clinicopathological correlation were noted. Histomorphology augmented by routine IHC and prognostic markers will help inform the disease status at the time of diagnosis and give clues on further management and close follow-up.

\section{Additional Information}

\section{Disclosures}

Human subjects: Consent was obtained or waived by all participants in this study. Animal subjects: All authors have confirmed that this study did not involve animal subjects or tissue. Conflicts of interest: In compliance with the ICMJE uniform disclosure form, all authors declare the following: Payment/services info: All authors have declared that no financial support was received from any organization for the submitted work. Financial relationships: All authors have declared that they have no financial relationships at present or within the previous three years with any organizations that might have an interest in the submitted work. Other relationships: All authors have declared that there are no other relationships or activities that could appear to have influenced the submitted work.

\section{References}

1. Gujral S, Agarwal A, Gota V, et al.: A clinicopathologic study of mantle cell lymphoma in a single center study in India. Indian J Pathol Microbiol. 2008, 51:315-22. 10.4103/0377-4929.42503

2. Klapper W: Histopathology of mantle cell lymphoma. Semin Hematol. 2011, 48:148-54 10.1053/i.seminhematol.2011.03.006

3. Aqil B, Triska G, Frater J, et al.: Immunophenotypic variations in mantle cell lymphoma and their impact on clinical behavior and outcome. Arch Pathol Lab Med. 2018, 142:1268-1274. 10.5858/arpa.2017-0368-OA

4. Campo E, Swerdlow SH, Harris NL, Pileri S, Stein H, Jaffe ES: The 2008 WHO classification of lymphoid neoplasms and beyond: evolving concepts and practical applications. Blood. 2011, 117:5019-5032. 10.1182/blood-2011-01-293050

5. Gao J, Peterson L, Nelson B, Goolsby C, Chen YH: Immunophenotypic variations in mantle cell lymphoma. Am J Clin Pathol. 2009, 132:699-706. 10.1309/AJCPV8LN5ENMZOVY

6. Zanetto U, Dong H, Huang Y, et al.: Mantle cell lymphoma with aberrant expression of CD10 . Histopathology. 2008, 53:20-29. 10.1111/j.1365-2559.2008.03060.x

7. Tapia G, Lopez R, Muñoz-Mármol AM, et al.: Immunohistochemical detection of MYC protein correlates with MYC gene status in aggressive B cell lymphomas. Histopathology. 2011, 59:672-678. 10.1111/j.13652559.2011.03978.x

8. Hernandez L, Fest T, Cazorla M, et al.: p53 gene mutations and protein overexpression are associated with aggressive variants of mantle cell lymphomas. Blood. 1996, 87:3351-3359.

9. Ribera-Cortada I, Martinez D, Amador V, et al.: Plasma cell and terminal B-cell differentiation in mantle cell lymphoma mainly occur in the SOX11-negative subtype. Mod Pathol. 2015, 28:1435-1447. 10.1038/modpathol.2015.99

10. Naresh KN, Agarwal B, Sangal BC, Basu DD, Kothari AS, Soman CS: Regional variation in the distribution of subtypes of lymphoid neoplasms in India. Leuk Lymphoma. 2002, 43:1939-1943. 10.1080/1042819021000016069

11. Hrgovic I, Hartmann S, Steffen B, Vogl T, Kaufmann R, Meissner M: Cutaneous involvement as a rare first sign of systemic mantle cell lymphoma: a case report and review of the literature. Mol Clin Oncol. 2016, 4:728-732. 10.3892/mco.2016.792

12. Carvajal-Cuenca A, Sua LF, Silva NM, et al.: In situ mantle cell lymphoma: clinical implications of an incidental finding with indolent clinical behavior. Haematologica. 2012, 97:270-278. 10.3324/haematol.2011.052621

13. Roy A, Kar R, Basu D: Nodal mantle cell lymphoma: a descriptive study from a tertiary care center in south India. Indian J Pathol Microbiol. 2013, 56:94-97. 10.4103/0377-4929.118680

14. Pérez-Galán P, Dreyling M, Wiestner A: Mantle cell lymphoma: biology, pathogenesis, and the molecular basis of treatment in the genomic era. Blood. 2011, 117:26-38. 10.1182/blood-2010-04-189977

15. Mozos A, Royo C, Hartmann E, et al.: SOX11 expression is highly specific for mantle cell lymphoma and identifies the cyclin D1-negative subtype. Haematologica. 2009, 94:1555-1562. 10.3324/haematol.2009.010264

16. Kwatra KS, Paul PA, Dhaliwal D, Calton N, John JM: Mantle cell lymphoma and variants: a clinicopathological and immunohistochemical study. Int J Sci Stud. 2016, 3:166-172. 
17. Miao Y, Lin P, Saksena A, et al.: CD5-negative mantle cell lymphoma: clinicopathologic correlations and outcome in 58 patients. Am J Surg Pathol. 2019, 43:1052-1060. 10.1097/PAS.0000000000001278

18. Shih AR, Bledsoe JR, McKelvie P, Louissaint A, Harris NL, Zuckerberg L: CD5-negative mantle cell lymphoma shows a less aggressive outcome and variable SOX11 staining. J Hematopathol. 2017, 10:49-53.

19. Saksena A, Yin CC, Xu J, et al.: CD23 expression in mantle cell lymphoma is associated with CD200 expression, leukemic non-nodal form, and a better prognosis. Hum Pathol. 2019, 89:71-80. 10.1016/j.humpath.2019.04.010

20. Xu J, Wang L, Li J, et al.: SOX11-negative mantle cell lymphoma: clinicopathologic and prognostic features of 75 patients. Am J Surg Pathol. 2019, 43:710-716. 10.1097/PAS.0000000000001233

21. Nygren L, Baumgartner Wennerholm S, Klimkowska M, Christensson B, Kimby E, Sander B: Prognostic role of SOX11 in a population-based cohort of mantle cell lymphoma. Blood. 2012, 119:4215-4223. 10.1182/blood-2011-12-400580

22. Fernàndez V, Salamero $\mathrm{O}$, Espinet $\mathrm{B}$, et al.: Genomic and gene expression profiling defines indolent forms of mantle cell lymphoma. Cancer Res. 2010, 70:1408-1418. 10.1158/0008-5472.CAN-09-3419

23. Gualco G, Weiss LM, Harrington WJ Jr, Bacchi CE: BCL6, MUM1, and CD10 expression in mantle cell lymphoma. Appl Immunohistochem Mol Morphol. 2010, 18:103-108. 10.1097/PAI.0b013e3181bb9edf

24. Martinez A, Pittaluga S, Rudelius M, et al.: Expression of the interferon regulatory factor 8/ICSBP-1 in human reactive lymphoid tissues and B-cell lymphomas: a novel germinal center marker. Am J Surg Pathol. 2008, 32:1190-1200. 10.1097/PAS.0b013e318166f46a

25. Hartmann E, Fernàndez V, Stoecklein H, Hernández L, Campo E, Rosenwald A: Increased MDM2 expression is associated with inferior survival in mantle-cell lymphoma, but not related to the MDM2 SNP309. Haematologica. 2007, 92:574-575. 10.3324/haematol.10891

26. Kanai R, Miyagawa-Hayashino A, Shishido-Hara Y, et al.: Mantle cell lymphoma with EBV-positive Hodgkin and Reed-Sternberg-like cells in a patient after autologous PBSCT: phenotypically distinct but genetically related tumors. Pathol Int. 2021, 71:96-101. 10.1111/pin.13038 